DOI: https://doi.org/10.14311/TPFM.2022.012

\title{
DEVELOPING A COUPLED CFD SOLVER FOR MASS, MOMENTUM AND HEAT TRANSPORT IN CATALYTIC FILTERS
}

\author{
Tomáš Hlavatý ${ }^{1,2}$, Martin Isoz ${ }^{1,2}$, Petr Kočí ${ }^{3}$ \\ ${ }^{1}$ Czech Academy of Sciences, Institute of Thermomechanics, Dolejškova 5, Prague 18200 , \\ Czech Republic \\ ${ }^{2}$ Department of Mathematics, Faculty of Chemical Engineering, University of Chemistry and \\ Technology, Technická 5, Prague 166 28, Czech Republic \\ ${ }^{3}$ Department of Chemical Engineering, Faculty of Chemical Engineering, University of Chem- \\ istry and Technology, Technická 5, Prague 166 28, Czech Republic
}

\begin{abstract}
Using catalytic filters $(\mathrm{CF})$ in automotive exhaust gas aftertreatment decreases the system heat losses and facilitates the $\mathrm{CF}$ regeneration. On the other hand, the $\mathrm{CF}$ overall performance is strongly dependent on the catalytic material distribution within it. In the present work, we aim to provide a computational framework to study the dependence of the CF characteristics, i.e. the pressure loss and the conversion of gaseous pollutants, on the catalyst distribution. Previously, we built an isothermal computational fluid dynamics (CFD) model of the flow and conversion of gaseous pollutants inside the $\mathrm{CF}$. However, the reactions occurring inside the $\mathrm{CF}$ are exothermic and the assumption of constant temperature proved to be too restricting for real-life applications of the developed isothermal CFD model. Thus, in this work, we extend the framework by the enthalpy balance, which requires combining all the transport equations (mass, momentum and enthalpy) in a single solver. The new and more general solver provides results in good agreement with a well established $(1+1) \mathrm{D}$ channel model calibrated on experimental data. Furthermore, it allows studying more complex device-scale geometries of laboratory CF samples.
\end{abstract}

Keywords: CFD, Catalytic filter, OpenFOAM, Gasoline particulate filter (GPF).

\section{Introduction}

The increasing number of vehicles and higher interest in ecology lead to tightening of laws on automotive exhaust gas after-treatment. Formerly, the automotive exhaust gas after-treatment comprised two groups of clean-up devices, (i) filters trapping the particulate matter (PM), and (ii) catalytic converters for conversion of environment endangering gases [1]. However, a recent trend is to combine these devices into a single one, a catalytic filter (CF) [2]. As depicted in Fig. 1, the catalytic filter is made of numerous parallel alternatively plugged channels in a honeycomb arrangement that are coated with the catalytic material. Consequently, CF is capable of removing both solid and gaseous pollutants.

Replacing the catalytic converters and PM filters with a single device is cheaper, decreases the system heat losses and facilitates the $\mathrm{CF}$ regeneration in the presence of the catalyst. On the other hand, the overall $\mathrm{CF}$ performance strongly depends on the catalytic material distribution that has to be carefully optimized [3]. The catalytic material distribution can vary (i) on the pore scale the deposition of the catalyst on or in the wall, c.f. Fig. 1 (right), and (ii) on the device scale the deposition of the catalyst within the whole device.

Hence, in order to correctly estimate the effects of the catalyst distribution on CF performance, a model resolving the CF pore and device scale is required. However, simultaneous solution of both these scales is computationally unfeasible. Thus, a multi scale modeling framework is needed. The idea behind such a framework is (i) to simulate flow and reaction in the pore-scale computational domain that is reconstructed using X-ray tomography for multiple samples from different locations in a real-life CF, (ii) based on the pore-scale results, estimate effective local wall properties, and (iii) use the effective local wall properties combined with the global device-scale catalyst distribution to estimate the $\mathrm{CF}$ process characteristics. Thus, a goal is to provide a fully three-dimensional CFD solver, resolving flow, species and enthalpy transport in an arbitrary geometry of the CF at an arbitrary scale.

Such a multi-scale CFD framework has been recently developed in our laboratory $[1,2,3]$. Up to now, we assumed the system to be isothermal on all the scales of interest. However, the reactions 


\section{Device scale}

Channel scale

Pore scale
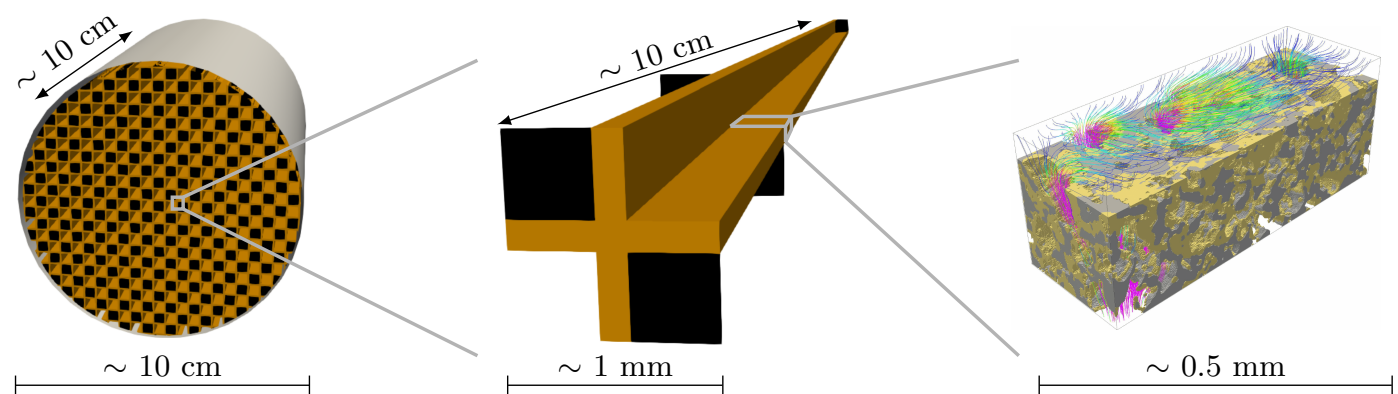

Figure 1: Complete monolithic catalytic filter (left), a representative elementary unit of a catalytic filter (center) and local pore-scale geometry (right).

occurring inside the $\mathrm{CF}$ are strongly exothermic and the assumption of the constant temperature, while it holds on the pore scale, has been proven too restricting for real-life applications of the device scale models. Hence, the main goal of present work is to extend the existing isothermal framework by an enthalpy balance. Such an extension (i) allows to keep the temperature variable in the domain and consequently modify the gas and material properties in dependence on it, but (ii) requires the momentum, mass, enthalpy and reactive species transport equations to be solved simultaneously.

In the present paper, we (i) introduce the new non-isothermal solver governing equations with an emphasis on linking the pore and the device scale, (ii) verify the new solver verification against a well established (1+1)D XPF model [4] that works at the channel scale, note Fig. 1 (center) and is calibrated on experimental data, and (iii) demonstrate the new solver capabilities on a laboratory device scale geometry, where we study the radial distribution of the temperature and carbon monoxide molar fraction within the device for the case of a $\mathrm{CF}$ with heat losses into the environment.

\section{Description of the developed model}

The new non-isothermal solver is built on the same balance principles as the isothermal solver presented in $[1,5]$. However, the heat released from exothermic reactions occurring inside the catalytic filter is taken into account and the temperature is assumed variable within the computational domain.

\subsection{Governing equations}

Overall, we solve the problem of non-isothermal incompressible flow of a reactive Newtonian fluid (the ideal gas) inside the catalytic filter geometry divided into free channels $\left(\Omega_{f}\right)$ and porous walls coated with a catalytic material $\left(\Omega_{w}\right.$, shown in orange in Fig. 1 (center)). The problem is solved under the steady-state assumption. Marking $\boldsymbol{u}$ the fluid velocity, $p$ pressure, $\mu$ dynamic viscosity, $y_{i}$ molar fraction of the $i$-th specie, and $T$ temperature, the model equations can be written as

$$
\begin{aligned}
\nabla \cdot(\rho \boldsymbol{u} \otimes \boldsymbol{u})-\nabla \cdot(\mu \nabla \boldsymbol{u}) & =-\nabla p+\rho s, \quad \rho=\frac{p M^{\mathrm{g}}}{\mathrm{R}^{\mathrm{g}} T}, \\
\nabla \cdot(\rho \boldsymbol{u}) & =0 \\
\nabla \cdot\left(\boldsymbol{u} c_{\mathrm{T}} y_{i}\right)-\nabla \cdot\left(D_{i}^{\mathrm{eff}} c_{\mathrm{T}} \nabla y_{i}\right) & =\nabla \cdot\left(D_{i}^{\mathrm{eff}} y_{i} \nabla c_{\mathrm{T}}\right)+r_{i}, \quad i=1, \ldots, n, \quad c_{\mathrm{T}}=\frac{\rho}{M^{\mathrm{g}}}, \\
\nabla \cdot\left(\rho \boldsymbol{u} c_{\mathrm{p}} T\right)-\nabla \cdot\left(\lambda^{\mathrm{eff}} \nabla T\right) & =s^{h},
\end{aligned}
$$

where the equations (1) represent the momentum and mass balance, respectively. Equation (2) stands for the $i$-th molar specie balance and equation (3) gives the enthalpy balance in the computational domain $\Omega=\Omega_{f} \cup \Omega_{w} \subset \mathbb{R}^{3}, \Omega_{f} \cap \Omega_{w}=\emptyset$.

Concentrating on the momentum and mass transport (1), note that the gas density $\rho$ is not an independent system variable as it is linked with $T$ and $p$ via the ideal gas state equation (1)right, where $M^{\mathrm{g}}$ is the molar mass of the flowing gas and $\mathrm{R}^{\mathrm{g}}$ is the universal ideal gas constant. 
Consequently, also the total molar concentration $c_{\mathrm{T}}$ is assumed variable in $\Omega$ and the molar balances for individual reactive species present in the exhaust gas can be written in a form given in (2) with the molar fractions $y_{i}, i=1, \ldots, n$ being the primary variables.

Finally, the enthalpy balance (3) is formulated with $T$ as the primary variable, all the mechanical heat sources neglected and the reference temperature set equal to zero $\left(T_{\text {ref }}=0{ }^{\circ} \mathrm{C}\right)$. Note that the specific gas heat capacity $c_{\mathrm{p}}$ and effective thermal diffusivity $\lambda^{\mathrm{eff}}$ are treated as temperaturedependent.

\section{$2.2 \quad$ Effective transport properties}

As stated above, the computational domain $\Omega$ is heterogeneous, meaning that it comprises free channels $\left(\Omega_{f}\right)$ and the porous walls $\left(\Omega_{w}\right)$. However, the whole domain is modeled as a single region and $\Omega_{w}$ is treated as a porous zone. Consequently, transport properties describing the media vary within $\Omega$. In the present work, the domain non-homogeneity is resolved by an introduction of effective transport physical properties.

In particular, focusing on the diffusivity computation, we calculate the free diffusivity coefficient of the $i$-th specie $D_{i}$ from the Fuller equation and use it in the $\Omega_{f}$, while in $\Omega_{w}$, we lower the free diffusion coefficient by the wall porosity $\varepsilon$ and tortuosity $\tau$ as indicated in $(4)_{1}$.

$$
D_{i}^{\mathrm{eff}}=\left\{\begin{array}{rl}
D_{i} & \text { in } \Omega_{f} \\
D_{i} \frac{\varepsilon}{\tau} & \text { in } \Omega_{w}
\end{array} \quad, \quad \lambda^{\mathrm{eff}}=\left\{\begin{aligned}
\lambda_{\text {gas }} & \text { in } \Omega_{f} \\
\varepsilon \lambda_{\text {gas }}+(1-\varepsilon) \lambda_{\mathrm{w}} & \text { in } \Omega_{w}
\end{aligned}\right.\right.
$$

Besides, note that the nitrogen is chosen as the reference gas for the diffusivity computations and the standard Fick diffusion is assumed.

Similarly as the effective diffusion coefficient, also the effective thermal diffusivity $\lambda^{\text {eff }}$ present in the enthalpy balance (3) is piece-wise continuous in $\Omega$ according to $(4)_{2}$. Note that we assume the effective thermal diffusivity $\lambda^{\text {eff }}$ to be a simple convex combination of the gas $\lambda_{\text {gas }}$ and wall $\lambda_{\mathrm{w}}$ thermal diffusivities in $\Omega_{w}$.

\subsection{Sources in used transport equations}

A link between the pore and device scale simulations is established by using an additional source term $\rho \boldsymbol{s}$ in the momentum equation $(1)_{1}$ in device and channel scale simulations. The term expresses the resistance to the flow in porous media, and it is computed utilizing the Darcy permeability model $(5)_{1}$, where $\kappa$ is the local Darcy permeability of the porous coated filter wall estimated from the pore scale CFD simulation results.

$$
\rho s=\left\{\begin{array}{rl}
\mathbf{0} & \text { in } \Omega_{f} \\
-\frac{\mu}{\kappa} \boldsymbol{u} & \text { in } \Omega_{w}
\end{array} \quad, \quad r_{i}=\left\{\begin{array}{rl}
0 & \text { in } \Omega_{f} \\
\varphi^{\mathrm{c}} \nu_{i} r^{\mathrm{c}} & \text { in } \Omega_{w}
\end{array}, \quad s^{h}=r_{\Delta} H^{r}\right.\right.
$$

Furthermore, the reaction is assumed to occur solely in the catalytic coating, which is present only in the CF porous walls. Therefore, the reaction term $r_{i}$ given in $(5)_{2}$ is active only in the porous walls, where $\varphi^{\mathrm{c}}$ is the volumetric fraction of the catalytic material in $\Omega_{w}$. Next, marking the $\nu_{i}$ stoichiometric coefficient and ${ }_{\Delta} H^{r}$ standard reaction enthalpy, the reaction heat source $s^{h}$ is defined in $(5)_{3}$. Note that due to the simplicity and conciseness of notation in $(5)_{2}$ and $(5)_{3}$, only a single reaction is assumed to occur in the system. However, the solver is prepared to be supplied with arbitrary kinetics.

\subsection{Notes on the model implementation}

The presented model was implemented within the OpenFOAM finite volume method (FVM) library [6]. The overall solution procedure is a segregated one. The solution of flow (1) is based on the standard SIMPLE algorithm and the individual specie balances (2) are solved sequentially. During the model development, it was found out that in each solver iteration it is profitable to first solve the momentum balance $(1)_{1}$, followed by the sequential solution of the reacting species molar balances (2) and of the enthalpy balance (3), and to finish the iteration by solving the pressure equation stemming SIMPLE pressure-velocity coupling. Furthermore both the reaction rate and heat source are linearized within the solver implementation in order to enhance solver convergence. 


\section{Solver verification and proof-of-concept application}

The new solver was tested on the case of the dominant chemical reaction occurring in the catalytic filters - carbon monoxide oxidation. Using the previous notation, the assumed reaction kinetics can be written as,

$$
\mathrm{CO}+\frac{1}{2} \mathrm{O}_{2} \rightarrow \mathrm{CO}_{2}, r^{c}=k \frac{y_{\mathrm{CO}} y_{\mathrm{O} 2}}{\left(1+K_{\mathrm{inh}} y_{\mathrm{CO}}\right)^{2}}, k=\mathrm{k}_{0} \exp \left(\frac{-\mathrm{E}_{\mathrm{a}}}{\mathrm{R}^{\mathrm{g}} T}\right), K_{\mathrm{inh}}=\mathrm{K}_{0} \exp \left(\frac{\mathrm{E}_{\mathrm{inh}}}{T}\right) .
$$

The temperature dependence of the kinetic and equilibrium constants is described by the Arrhenius law and values of the pre-exponential factors and activation energies can be found in [1].

\subsection{Numerical settings and convergence}

Before the solver verification, we present a characteristic simulation numerical setup, and a typical solver convergence behavior. Note that although the solver itself may be used with arbitrary turbulence models implemented in the OpenFOAM library, only laminar flows are of interest in the studied applications. The individual terms in equations $(1,2,3)$ were discretized as follows, (i) the convective terms $\left(\nabla \cdot(\rho \boldsymbol{u} \otimes \phi), \phi=\left\{\boldsymbol{u}, y_{i}, T\right\}\right)$ were discretized using bounded Gauss linearUpwind OF scheme, (ii) the diffusion terms $\left(\nabla \cdot(\Gamma \nabla \phi), \Gamma=\left\{\mu, D_{\mathrm{i}}^{\text {eff }}, \lambda^{\text {eff }}\right\}\right)$ were discretized with Gauss linear limited corrected $0.5 \mathrm{OF}$ scheme, and (iii) interpolation from cell to cell face centroids was performed as linear. Note that more information on the specific schemes can be found for example in [7].

\begin{tabular}{ccc}
\hline & $\alpha_{f}$ & $\alpha_{e}$ \\
\hline$p$ & 0.1 & 0.95 \\
$\boldsymbol{u}$ & 0.2 & 0.95 \\
$T$ & 1 & 0.98 \\
$y_{i}$ & 1 & 0.98 \\
\hline
\end{tabular}

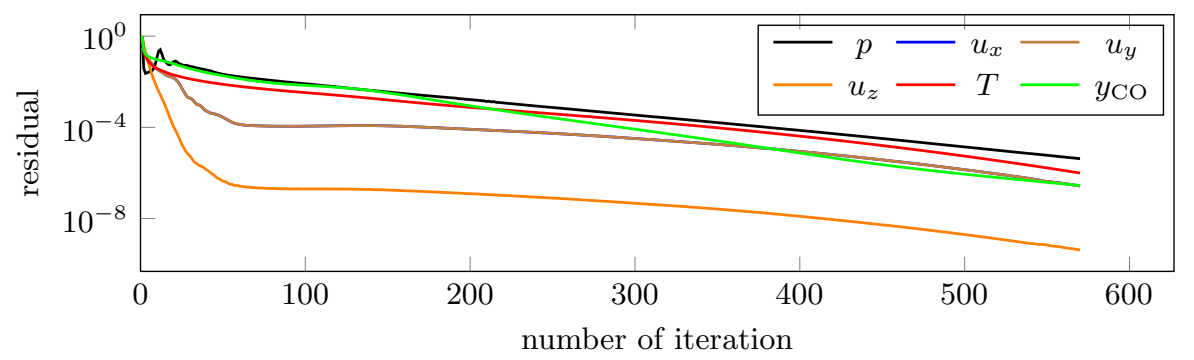

Figure 2: OpenFOAM relaxation criteria (left), and example of the solution convergence (right).

In Fig. 2, we show the solution convergence in the channel scale geometry and give relaxation factors for individual fields and corresponding equations as implemented in OpenFOAM. Note that the overall solver convergence is as expected from a segregated solution of Navier-Stokes equations for a laminar flow. Consequently, the implemented coupling between the transport equations does not deteriorate the qualitative convergence behavior, e.g. no oscillations in the residuals were observed for the shown case, which is discretized on a structured hexahedral mesh.

\subsection{Solver verification}

For a simplified geometry comprising four quarter-channels of the catalytic filter and leveraging the available symmetries, as shown in Fig. 1 (center), it is possible to compare the results of the new CFD solver to a an established yet simplified $(1+1) \mathrm{D}$ XPF solver, which was calibrated on experimental data [4].

Ignition curve Firstly, we compare the ignition curve, i.e. the dependence of the average outlet molar CO fraction on the inlet temperature. The results for the old isothermal CFD model, new non-isothermal CFD model, and isothermal and non-isothermal variants of the XPF model are given in Fig. 3. It may be observed that for both the isothermal and non-isothermal computations, the CFD results are in a good agreement with the XPF model. Furthermore, the non-isothermal models provide a significantly lower ignition temperature $\left(\sim 205^{\circ} \mathrm{C}\right)$ than the isothermal ones $\left(\sim 225^{\circ} \mathrm{C}\right)$. Such a behavior is to be expected because the considered reaction $(6)$ is exothermic and its reaction rate $r^{c}$ increases with temperature. Consequently, the CF heating facilitates the ongoing reaction and lowers the ignition temperature. 


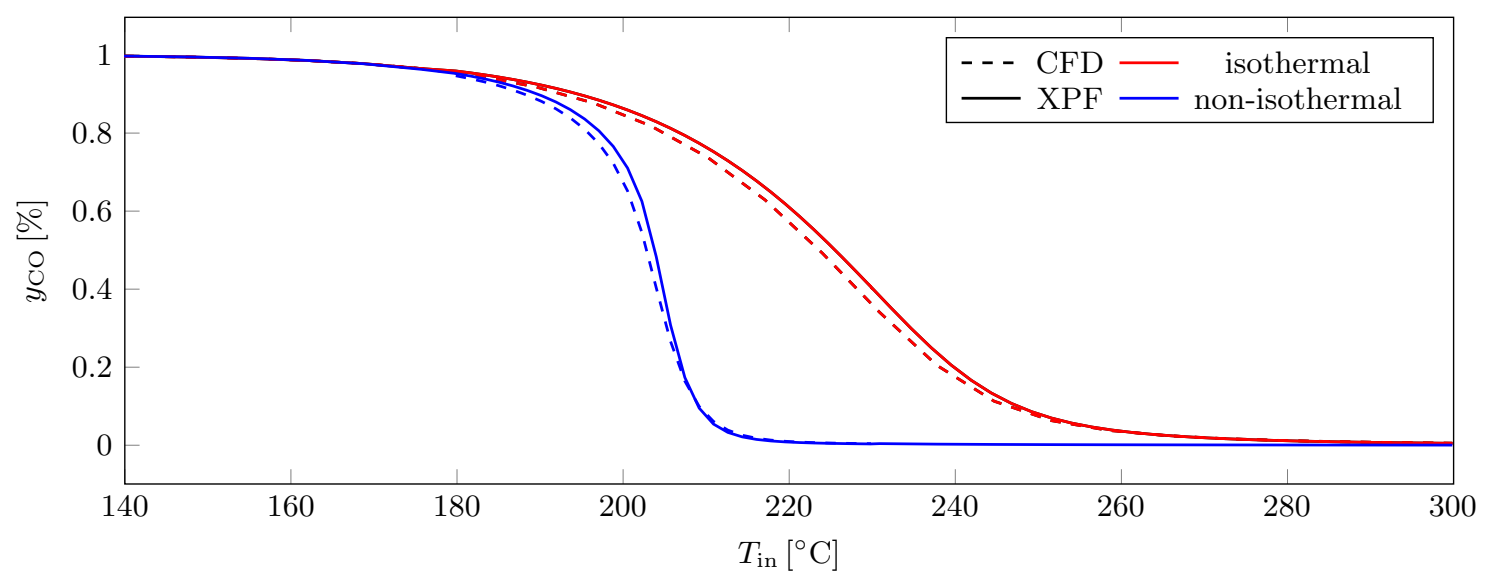

Figure 3: Ignition curves for isothermal and non-isothermal variants of the both CFD and XPF models $\left(u_{\mathrm{in}}=1.6 \mathrm{~m} \mathrm{~s}^{-1}, y_{\mathrm{CO}, \text { in }}=1 \%\right)$.
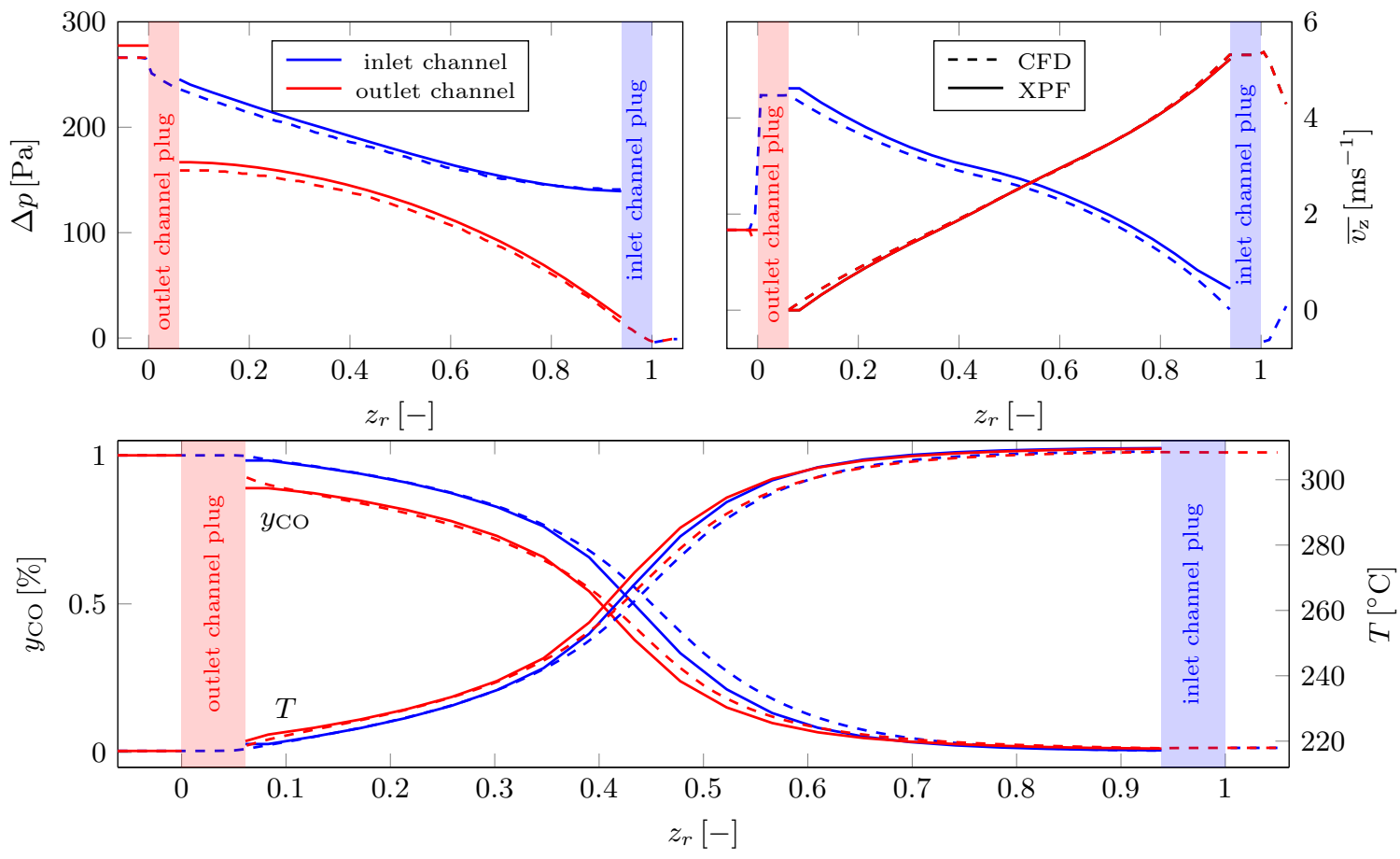

Figure 4: Pressure, velocity streamwise component, temperature and $\mathrm{CO}$ molar fraction profiles for the new CFD and XPF models $\left(u_{\mathrm{in}}=1.67 \mathrm{~m} \mathrm{~s}^{-1}, T_{\mathrm{in}}=217^{\circ} \mathrm{C}, y_{\mathrm{CO}, \text { in }}=1 \%\right)$. 
Data profiles along the $\mathbf{C F}$ channels Next, for a selected inlet temperature of $T_{\text {in }}=217^{\circ} \mathrm{C}$, we evaluate the pressure, streamwise velocity, $\mathrm{CO}$ molar fraction and temperature profiles along the CF channels. A verification of the non-isothermal CFD model results against the XPF is given in Fig. 4. The profiles of all the variables are in good agreement in both the inlet and outlet CF channels. This is a significant achievement especially with respect to the pressure since inlet effects originating in changes in the flow occurring between the inlet buffer and the inlet channels are inherently present in the 3D CFD simulations but in XPF, they need to be added based on empirical correlations.
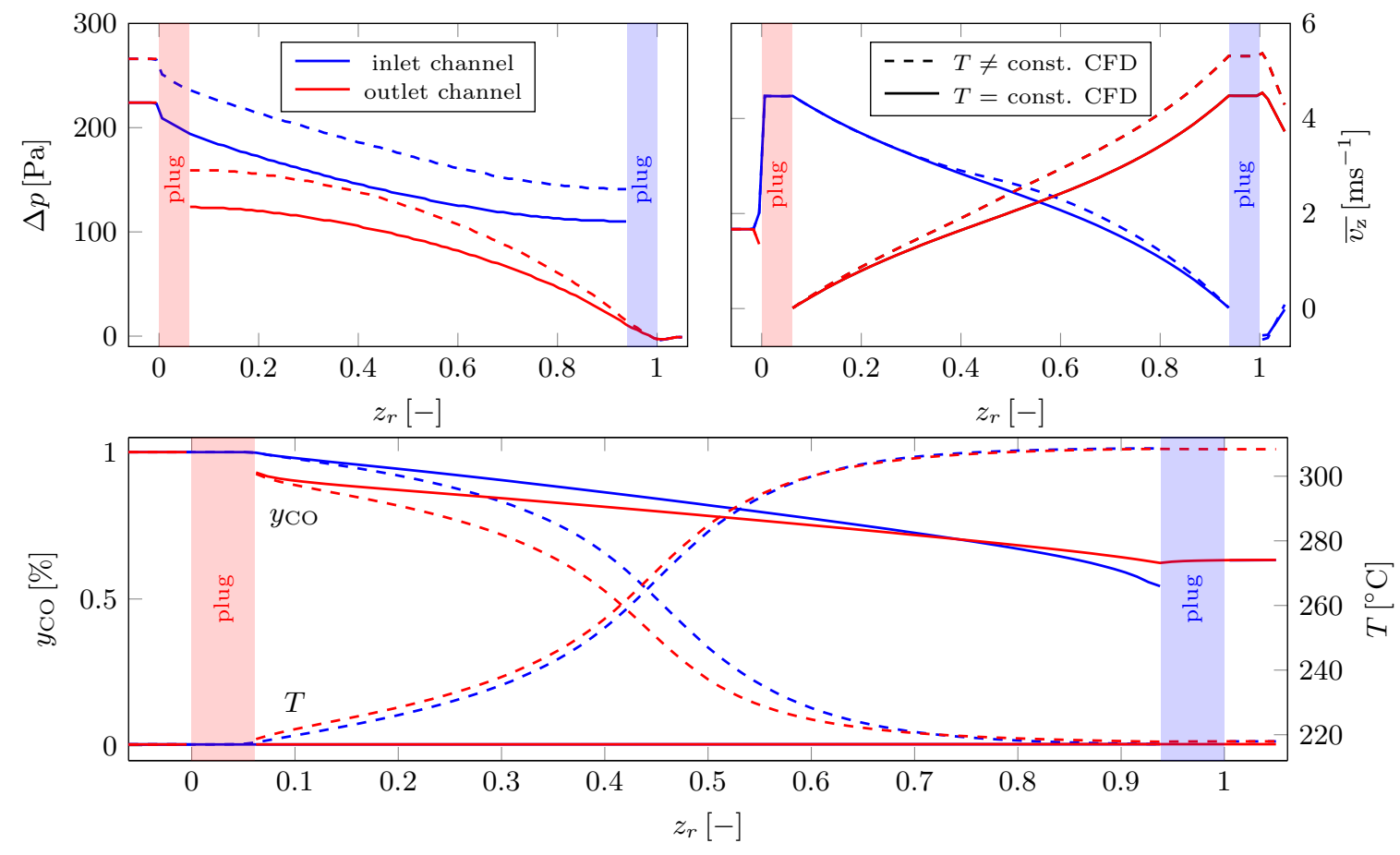

Figure 5: Pressure, velocity streamwise component, temperature and CO molar fraction profiles for the isothermal and non-isothermal CFD model $\left(u_{\mathrm{in}}=1.67 \mathrm{~m} \mathrm{~s}^{-1}, T_{\text {in }}=217^{\circ} \mathrm{C}, y_{\mathrm{CO} \text {,in }}=1 \%\right)$.

Furthermore, in Fig. 5, we compare the old isothermal and new non-isothermal CFD models to show the necessity of taking the channel and device scale temperature variations into account. Note that to obtain similar results from the isothermal and non-isothermal models the CO inlet molar fraction needs to be of order of $0.1 \%$ at most. However, the CO molar fractions encountered in the automotive exhaust gas often exceed $1 \%$. For the $y_{\mathrm{CO}, \text { in }}=1 \%$ and the tested temperature of $T_{\text {in }}=217^{\circ} \mathrm{C}$, there are significant discrepancies between the isothermal and non-isothermal model results. Particularly, the pressure loss of the device increases in the non-isothermal case as the dynamic viscosity of the gas increases with the increasing temperature. Furthermore, considering streamwise velocity component, note that in the isothermal case, velocity at the beginning of the inlet channel is same as at the end of the outlet channel, while in the non-isothermal case, velocity at the end of the outlet channel is higher because of the thermal expansion.

Nevertheless, the most pronounced discrepancy between the two models is encountered in the $\mathrm{CO}$ conversion estimates. As already discussed when describing the ignition curve, the temperature has an autocatalytic effect on the considered reaction. The selected $T_{\text {in }}=217^{\circ} \mathrm{C}$ falls within the region in which the non-isothermal model estimates an already ignited CO mixture and the isothermal model does not. Consequently, the non-isothermal model predicts a complete CO conversion along the CF while the isothermal one estimates that only $35 \%$ of CO is converted.

\subsection{Device scale results for a laboratory catalytic filter sample}

The generality of the new solver is shown on a case of a device scale simulation. With respect to symmetry, the computational domain is constructed as one quarter of a device comprising in total 
twenty channels across its diameter, see Fig. 6. Such a geometry and the used inlet velocity of $u_{\text {in }}=1.67 \mathrm{~m} \mathrm{~s}^{-1}$ are inspired by a common experimental setup, e.g. [8]. In order to show the 3D solver capabilities, we study (i) an adiabatic case, Fig. 6a, and (ii) a case of the CF with a heat loss to the environment (non-adiabatic), Fig. $6 \mathrm{~b}$.

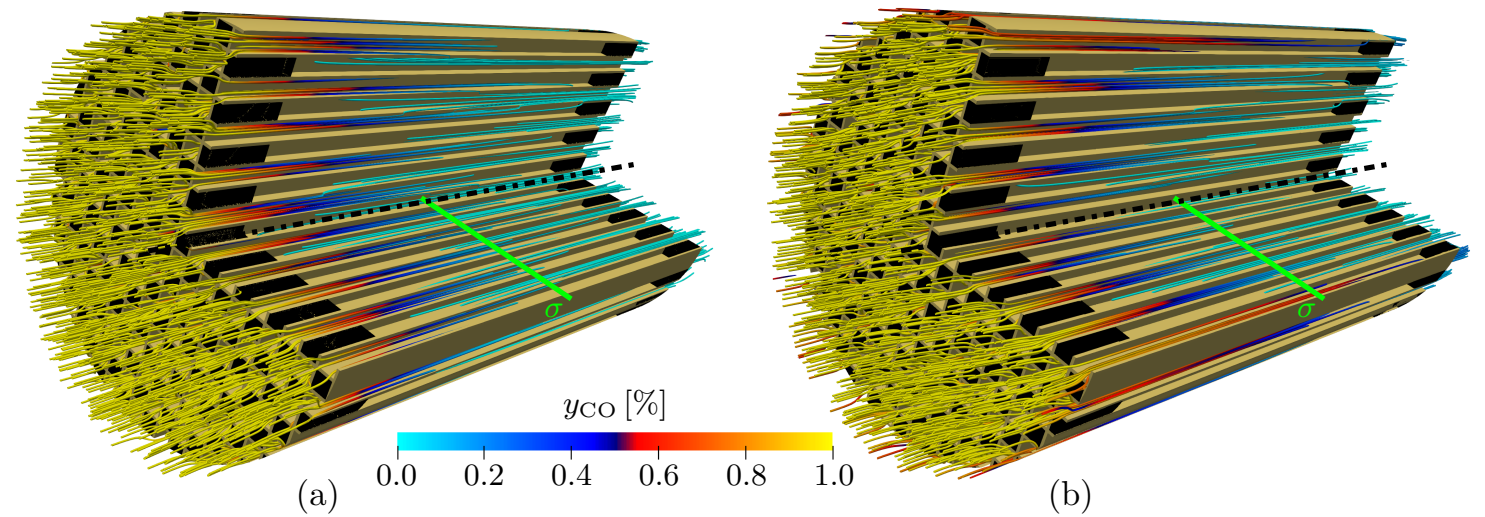

Figure 6: Laboratory device scale geometry with streamlines colored by CO molar fraction. (a) Adiabatic, and (b) non-adiabatic case $\left(u_{\mathrm{in}}=1.67 \mathrm{~m} \mathrm{~s}^{-1}, T_{\mathrm{in}}=300^{\circ} \mathrm{C}, y_{\mathrm{CO}, \mathrm{in}}=1 \%\right)$. Note the green line $\sigma$ and the black dashdotted line marking the symmetry planes intersection.

Focusing on the discrepancies between adiabatic and non-adiabatic results in Fig. 6, note that (i) CO molar fraction in inner channels is quantitatively comparable in both cases, but (ii) in channels on the sides of the device, the conversion of the CO is significantly lower in the nonadiabatic case. Such a situation results in overall lower CO conversion within the whole device, if simulated as non-adiabatic. Despite the high inlet temperature $T_{\text {in }}=300^{\circ} \mathrm{C}$ (c.f. Fig 3 ), the non-adiabatic $\mathrm{CO}$ conversion $\approx 95 \%$, while in the adiabatic case, it is complete.

Even better insight into the device scale results can be achieved looking onto radial distributions of the temperature $(T)$ and $\mathrm{CO}$ molar fraction $\left(y_{\mathrm{CO}}\right)$ in the device. In the left hand side of Fig. 7, we show longitudinal slices through the device colored by $T$ and $y_{\mathrm{CO}}$. In the figure right hand side, the radial profiles on line $\sigma$ for both the adiabatic and non-adiabatic case are depicted. Considering adiabatic case results, the radial profiles on the line $\sigma$ are, apart from the small imperfections caused by the device geometry, periodic for both variables. In the non-adiabatic case, the temperature on the sides is relatively low, which results in the above discussed low CO conversion.

\section{Conclusion}

An already existing isothermal framework resolving the flow and conversion of gaseous pollutants in the catalytic filter in automotive exhaust gas aftertreatment was extended by the enthalpy balance. This extension forced a complete rewriting of the solver and coupling all the governing equations in a single code. On the other hand, allowing temperature variations inside the catalytic filter enables a solution of cases with real-life operational parameters in the device scale. For the case of a simplified channel scale geometry, (i) the new solver was verified against results of the established XPF model, which is calibrated on experimental data, and (ii) a necessity of the non-isothermal approach was shown on a comparison of results of the isothermal and non-isothermal models. Furthermore, the new 3D solver capabilities were illustrated on a simulation of a non-adiabatic laboratory scale catalytic filter consisting of several channels.

\section{Acknowledgment}

The work was supported by the Grant project with No. GA19-22173S of the Czech Science Foundation, within institutional support RVO:61388998 and by the Centre of Excellence for Nonlinear Dynamic Behaviour of Advanced Materials in Engineering CZ.02.1.01/0.0/0.0/15_003/0000493 (Excellent Research Teams) in the framework of Operational Programme Research, Development and Education. 
a)
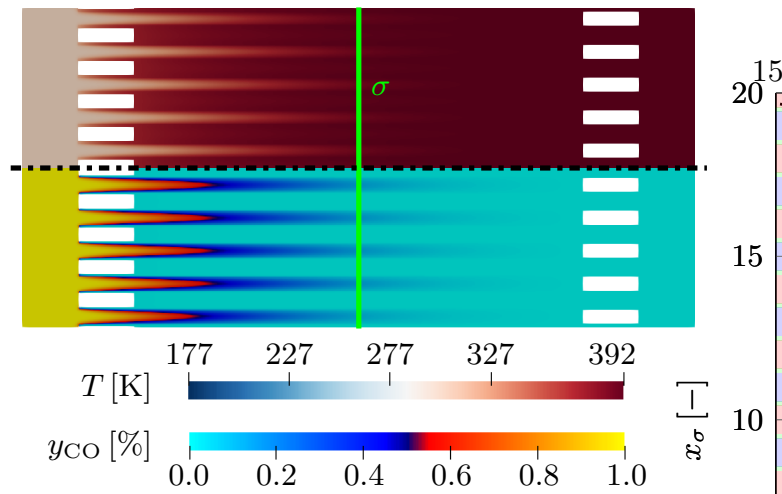

b)
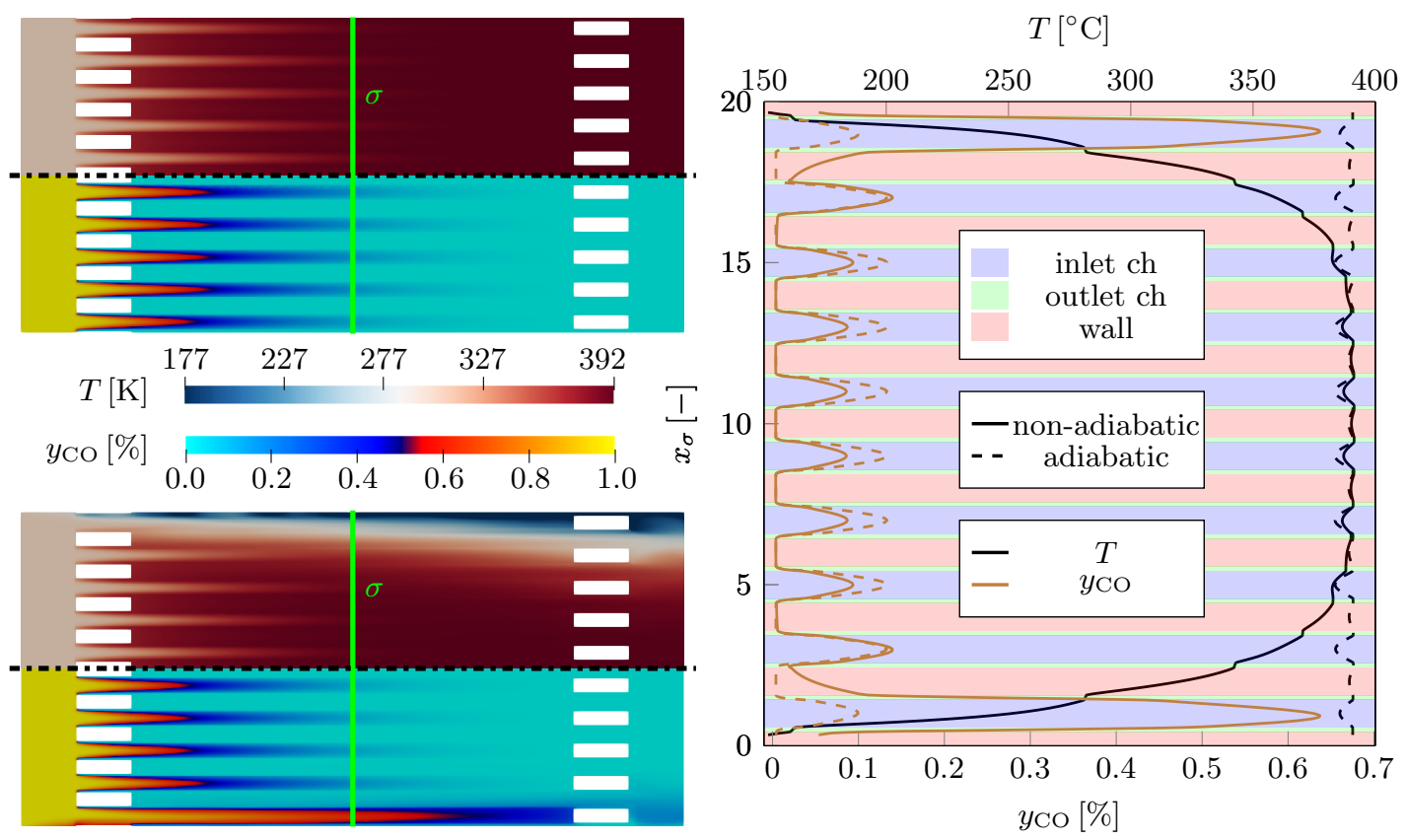

Figure 7: Longitudinal slices through the geometry colored by temperature and CO molar fraction (left), radial profiles of the temperature and CO molar fraction on the line $\sigma$ (right) for adiabatic case (a), and the case assuming the heat loss (b).

\section{References}

[1] Kočí, P., Isoz, M., Plachá, M., Arvajová, A., Václavík, M., Svoboda, M., Price, E., Novák, V. \& Thompsett, D.: 3D reconstruction and pore-scale modeling of coated catalytic filters for automotive exhaust gas aftertreatment. Cat. Today. vol. 320: (2019). pp. 165-174.

[2] Plachá, M., Kočí, P., Isoz, M., Svoboda, M., Price, E., Thompsett, D., Kallis, K. \& Tsolakis, A.: Pore-scale filtration model for coated catalytic filters in automotive exhaust gas aftertreatment. Chemical Engineering Science. vol. 226: (2020). page 115854.

[3] Leskovjan, M., Němec, J., Plachá, M., Kočí, P., Isoz, M., Svoboda, M., Novák, V., Price, E. \& Thompsett, D.: Multiscale modeling and analysis of pressure drop contributions in catalytic filters. Industrial \& Engineering Chemistry Research. vol. 60 no. 18: (2021). pp. 6512-6524.

[4] Schejbal, M., Štěpánek, J., Marek, M., Kočí, P. \& Kubíček, M.: Modelling of soot oxidation by $\mathrm{NO}_{2}$ in various types of diesel particulate filters. Fuel. vol. 89: (2010). pp. 2365-2375.

[5] Hlavatý, T., Isoz, M., Plachá, M., Šourek, M. \& Kočí, P.: Geometrically realistic macro-scale model for multi-scale simulations of catalytic filters for automotive gas aftertreatment. In Proceedings of the conference Topical Problems of Fluid Mechanics: pp. 82-89. Institute of Thermomechanics of the Czech Academy of Sciences: (2020).

[6] OpenCFD. OpenFOAM: The Open Source CFD Toolbox. User Guide. OpenCFD Ltd, UK: (2016).

[7] Moukalled, F., Darwish, M. \& Mangani, L. The finite volume method in computational fluid dynamics: an advanced introduction with OpenFOAM and Matlab. Springer-Verlag: Berlin, Germany: 1 edition: (2016). ISBN 978-3-319-16874-6.

[8] Mráček, D., Kočí, P., Marek, M., Choi, J.-S., Pihl, J. A. \& Partridge, W. P.: Dynamics of $\mathrm{N}_{2}$ and $\mathrm{N}_{2} \mathrm{O}$ peaks during and after the regeneration of lean $\mathrm{NO}_{x}$ trap. Applied Catalysis B: Environmental. vol. 166-167: (2015). pp. 509-517. 